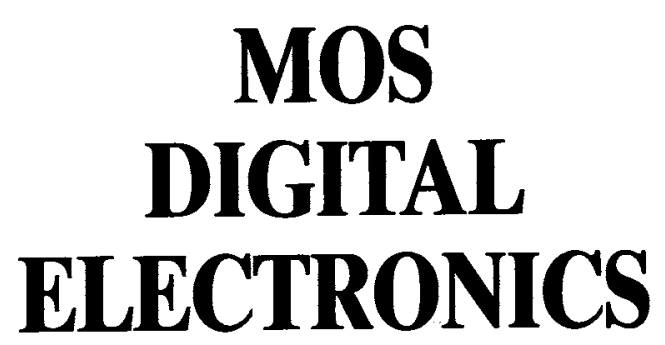


This page is intentionally left blank 


\section{MOS DIGITAL ELECTRONICS}

Stephen Shao-Chung Cheng 


\section{Published by}

World Scientific Publishing Co. Pte. Ltd.

P.O. Box 128, Farrer Road, Singapore 9128

U.S.A. office: World Scientific Publishing Co., Inc.

687 Hartwell Street, Teaneck NJ 07666, USA

Library of Congress Cataloging-in-Publication data is available.

\section{MOS DIGITAL ELECTRONICS}

Copyright $\odot 1987$ by World Scientific Publishing Co Pte Ltd.

All rights reserved. This book, or parts thereof, may not be reproduced in any form or by any means, electronic or mechanical, including photocopying, recording or any information storage and retrieval system now known or to be invented, without written permission from the Publisher.

ISBN 9971-50-372-7

Printed in Singapore by Fong and Sons Printers Pte. Ltd. 


\section{PREFACE}

As a result of the incredible progress in micro-electronics, the sizes of MOS transistors have been fast approaching physical limits. The feature size has become comparable to the diffraction limit of the visible light, commonly used in the making or processing of the tiny integrated circuits. The complexities of electronic systems that can be put or integrated on a single solid-state chip have also been increased to the point that it is hard to test and verify the product circuit. But once the reliable circuit or its solid-state chip is made, it is of tremendous usefuiness, efficiency and economy. On the other hand, the making of MOS circuits has reached an orderly maturity, in spite of the fact that any real progress is tedious and difficult. Needless to say that there is a wealth of technical information in forms of books and journals, which describe systematically the digital MOS circuits from the basic physics of devices, logic to large systems and their fabrication processes.

Digital electronics involves discreet signals and events, precisely dictated by appropriate logic functions. Fundamentally all complex functions are built upon a few elementary simple logic. Solid state circuits perform logic as continuous physical changes, which represent and result in corresponding designated discreet stable logic levels. It is the purpose of this book to take the approach that emphasizes the physical changes, while carefully keeping track on how the digital functions result.

It begins with the basic necessary physical concepts for the single MOS transistor, and shows how each part of the transistor works together to give continuous outputs, as the current-voltage equation of Ids approximates. The operations of various components of the inverter in the NMOS or CMOS are described in different voltage-current regions. These give then a total picture of the inverters, whose configuration is the starting points of the MOS logic family.

A commonly used jargon in describing the solid state circuitries through their evolution to smaller dimensions is scaling. For it, there are many new physical and practical problems that need to be studied in detail. Some essential characteristics remain in the current-voltage relation of the MOS transistor. The considerations of the scaling with respect to sizes, resistances and voltages as well 
as characteristic time constants are treated in a unified equation for MOS devices (transistors and inverters) for each of its sub-family with various clever schemes and load devices.

The designs of the MOS logic family are flexible. They do not necessarily need a fixed configuration in terms of circuit structures and layout. This book presents many examples of basic logic circuits and common logic functions, as they are realized in the MOS families. The complex logic functions therefore can be constructed in many ways to achieve minimum circuit complexities and areas and maximum speed and power saving. Using this freedom, this book further emphasizes the advantage in building clocked synchronous systems or logic state machines. The central roles of efficient simple clocked data registers and highly optimized logic are demonstrated. Many basic clocked logic devices are introduced. Some of them are described as simple finite state logic machines.

The usual devices with enhancement or depletion loads consume considerable power, because the load devices may be on or current carrying most of the time, while the driver devices are on half of the time on the average. Considerable amount of power can be saved if the load device can shut off for a large fraction of the time. For such purpose, the (Complementary MOS) CMOS family of logic circuitry is introduced. The driver group and load group or devices are seldom on at the same time. A current logic scheme is used for the finding and determination of the complementary nature of the CMOS logic devices. For a clocked CMOS system, further circuit saving scheme can be used, so the number of load devices are reduced to a minimum. Again the use of the current logic is extended to cover the operation of this case.

The role of all capacitances in a MOS circuit is critical. It stores the charges and affects the speed of the circuits, as it charges and discharges. The book discusses several topics, concerning the physical effects of the capacitances. Its effects can be minimized to make the circuit faster. It can also be utilized to perform many unusual operations.

For large and more complex logic systems, one would need to build more powerful MOS transistors, more organized ways to get the minimized logic and states. This is especially important and necessary in the modern computerized design environments. In the second half of this book, formal ways to achieve such goals is introduced. And the methods so developed can be put into the computer for large tasks for automatic and systematic solutions, as they are commonly carried out.

With all the basic elements understood and defined, one then can proceed 
to consider larger logic systems. The starting point is the finite state machine. The state machines are more complex and have the ability to make choices of next steps or decisions based on the present state of the machine as represented by the outputs and the present state of the input variables. They are realized by devices such as programmable logic arrays or programs stored in memory. The commands and communications among different logic machines or components are carried out or linked on buses.

The book also presents the ways the circuitry and systems can be described on paper and particularly by softwares. The designs and their descriptions therefore may resemble software programming. The information is organized to provide connectivity information and layout of circuitry over the two-dimensional chip area. The connectivity is contained in functional descriptions with associated input and output logic variables. When this is realized in actual layout, the physical structures have many processing layers, which are summarized in representative symbols. The symbols are relatively few; they provide an efficient method of descriptions.

There are many layout styles; they are optimized in different specific applications and design requirements. For example, the gate-array is used for relatively simple circuitry; standard cells can provide fast turn-around and efficient logic building blocks; and fully custom-design could give optimized circuitry for the entire system. Simple rules for layout are given, as examples, for general considerations.

Finally, one must know whether the design can work as expected. This is related to the problem of simulations and debugging. Present design systems and methodologies rely on software descriptions of the intended circuits to alleviate complexities and inflexibilities of the making of actual model of the circuitry, before the final solid-state chip is made. The modifications are then easier. With the circuit descriptions, one can simulate and test the circuit to gain quite complete understanding of the design. The book discusses and introduces two major extreme kinds of software simulators: logic and physical. Other intermediate kinds may have some advantages of each.

This book assumes background courses in elementary electricity and differential equations. It really requires good grasp of the physical contents from the mathematical equations and the circuitry. The second half of the book requires some knowledge of discreet mathematics and computer programming to appreciate fully the aims of the various presentations, which lead to automation of error free designs. The concepts are developed with actual basic 


\section{CONTENTS}

PREFACE

P.1

CHAPTER ONE

PHYSICAL DESCRIPTIONS OF MOS

TRANSISTORS

$1-1$

1.1 Resistive Model of the MOS-Inverter

$1-1$

1.1.1 Voltage Divider Relation for the Inverter

1.1.2 The Time-Characteristic of the Resistive Model

1.1.3 Consideration of the CMOS Inverter

1.2 Basic Physics of the Switching MOS

Transistor

1.6

1.2.1 MOS Transistor

1.2.2 Derivation of $\mathrm{lc}$ in Terms of Capacitances

1.2.3 Physical Explanation of the Current Equation

1.2.4 A More Accurate Approximation to the Channel Current Ids

1.2.5 Transistor Saturation Operation

1.2.6 Summary of Formulae and Parameters

1.3 Operational Parameters of MOS

Transistors

1.3.1 Effective MOS Channel

Resistance

1.3.2 Amplification Factor of the MOS Transistor

1.3.3 Inherent RchCg of MOS

Transistor 
1.3.4 Estimate of the RC Time Constant

1.3.5 The Threshold of a MOS

Transistor

1.4 Transistor Switching and Driving

Capability

1.4.1 Transistor Size Ratio

1.4.2 Relation of Minimum Gate Delay and Fanout

1.4.3 Power Consumption of the MOS Device

LOAD AND DRIVER DEVICES OF MOS CIRCUITS

2.1 Load Devices of MOS Circuits

2.1.1 The Enhancement Mode Load Devices

2.1.2 The Depletion Load Devices

2.1.3 The Load of the CMOS Devices

2.2 Voltage and Current Characteristics of MOS Inverters

2.2.1 Inverter with a Resistive Load

2.2.2 MOS Inverters with an Enhancement Load

2.2.3 MOS Inverters with a Depletion Load

2.2.4 CMOS Inverter

2.3 General Considerations of Scales of the MOS Devices

2.4 Time Dependence of the Switching MOS Inverters 
3.2 Logic Equations and Reductions

3.2.1 Logic Laws

3.2.2 Logic Equations and Their Reductions

3.2.3 Conversion to NOR - Gate only

3.2.4 Example of the Exclusive-OR Circuit

3.3 Simple Applications of Logic Devices

3.3.1 Turning on of Logic States or Paths

3.3.2 Simple Applications of Signal Controls

4.1 The Clocked Digital Systems

4.1.1 The D-Flip-Flops (Registers)

4.1.2 Static Storage Devices

4.1.3 Dynamic Storage Devices

4-8

4.1.4 Edge Triggered Devices

4.2 Basic Clocked Logic Units
4.2.1 Introduction to Logic State Machines
4.2.2 Simple State Machines as Building Blocks

\subsection{Shift Register and Time Decays}

5.4 A Simple State Machine that Recognizes Start-Code

5.4.1 Description

5-8

5.4.2 Definition of the Required States 
5.4.3 Extraneous State Assignments

5-11

5.4.4 Choices of the State Assignments

5.4.5 An Alternate J-K FF Implementation

5-12

$5-15$

5.5 Adder Circuitry

5-15

5.5.1 Binary Number System

5-15

5.5.2 Addition of Two Numbers

5.5.3 Logic Simplification of a Full Adder

5.5.4 Special Exclusive-OR MOS Circuitry

5.6 General CMOS Logic Devices

5.6.1 Basic Logic Devices

5.6.2 The Clocked CMOS Circuits

5-23

5.6.3 General Dynamic Logic Functions

5-25

5.7 Sequencer

5-28

5.8 Serial Memory
CHAPTER SIX

CHAPTER SEVEN
GENERAL CAPACITIVE PROPERTIES OF MOS CIRCUITS

6-1

6.1 Current Voltage Relation

6-1

6.2 Parasitic and Miller Capacitive Effect

6-2

6.3 Super Buffer Inverters

6-3

6.4 Use of Large Capacitances in MOS Circuits

6.6

6.4.1 The Basic Scheme

6-6

6.4.2 A Simple Application to Inverter

6-9

6.4.3 A Clocked Bootstrapped Driver

6-10

6.5 Gate Capacitances of MOS Transistors

FORMAL THEORY OF LOGIC, STATE EQUATIONS, AND REDUCTIONS

7.1 Representation of Logic Functions

7.2 Reductions of Minterms $7-2$

7.3 Reduction of Logic Functions 7-6 
7.4 Computer Notation of Prime Implicants $\quad 7-7$

7.5 Examples of Logic Reductions 7-7

7.6 Reductions of Multiple Output Logic

Functions

CHAPTER EIGHT

LOGIC STATE AND PLA

8-1

8.1 Logic State Reduction

8-1

8.1.1 Statement of the State Reduction Problem

8-1

8.1.2 Basic Concept of Relation

8-3

8.1.3 Applications of Equivalence Classes of States

8-5

8.2 Programmable Logic Array (PLA)

8-10

8.2.1 General Circuit Considerations

8-10

8.2.2 PLA Array

8-10

8.2.3 Area Saving of the PLA

8-13

CHAPTER NINE

DEVICES AND STORED-PROGRAM

MACHINES

9-1

9.1 State Machines

9-1

9.2 Nested State Machines

9-3

9.3 Memory Systems

9-5

9.3.1 ROM Units

9-6

9.3.2 Random Access Memory

9-7

9.4 Stack Operations

9-14

9.5 Communications and Controls over Bus

9.16

CHAPTER TEN

CIRCUIT DESCRIPTION AND LAYOUT

10-1

10.1 Circuit Description and Connectivity

10-1

10.2 Fanouts of Signals

10-3

10.3 Circuit Layout

10-3

10.3.1 Cell Layout

$10-4$

10.3.2 Design Rules and Symbolic

Layout

10.6 
10.3.3 Layout Examples and

Optimizations

10-7

10.4 Summary of Transistor Layout

10-23

CHAPTER ELEVEN TESTING AND VERIFICATION OF DESIGNS

11.1 Introduction

11-1

11.2 Definition of a Digital Circuit

$11-1$

11.3 Tests and Simulations

11-2

11.3.1 Circuit Model Building

11-2

11.3.2 Classes of Software Simulators

11-3

11.4 Test Strategies and Methods

11-5

11.4.1 Functional and Fault

Coverage Tests

11-5

11.4.2 Fault Coverage

11-5

11.4.3 Break Point Tests

$11-7$

11.5 Critical Paths and Debugging

11.11

REFERENCES

R.1

INDEX

I-1 